ISSN 1855-3966 (printed edn.), ISSN 1855-3974 (electronic edn.)

ARS MATHEMATICA CONTEMPORANEA 19 (2020) 337-349

https://doi.org/10.26493/1855-3974.2022.44a

(Also available at http://amc-journal.eu)

\title{
Properties of double Roman domination on cardinal products of graphs*
}

\author{
Antoaneta Klobučar \\ Faculty of Economics, University of Osijek, 31000 Osijek, Croatia
}

Ana Klobučar

Faculty of Mechanical Engineering and Naval Architecture, University of Zagreb, 10000 Zagreb, Croatia

Received 14 June 2019, accepted 25 August 2020, published online 20 November 2020

\begin{abstract}
Double Roman domination is a stronger version of Roman domination that doubles the protection. The areas now have $0,1,2$ or 3 legions. Every attacked area needs 2 legions for its defence, either their own, or borrowed from 1 or 2 neighbouring areas, which still have to keep at least 1 legion to themselves. The minimal number of legions in all areas together is equal to the double Roman domination number.

In this paper we determine an upper bound and a lower bound for double Roman domination numbers on cardinal product of any two graphs. Also we determine the exact values of double Roman domination numbers on $P_{2} \times G$ (for many types of graph $G$ ). Also, the double Roman domination number is found for $P_{2} \times P_{n}, P_{3} \times P_{n}, P_{4} \times P_{n}$, while upper and lower bounds are given for $P_{5} \times P_{n}$ and $P_{6} \times P_{n}$.

Finally, we will give a case study to determine the efficiency of double protection. We will compare double Roman domination versus Roman domination by running a simulation of a battle.
\end{abstract}

Keywords: Roman domination, double Roman domination, cardinal products of graphs, paths, cycles.

Math. Subj. Class. (2020): 05C69, 68U20

*This work has been fully supported by the Croatian Science Foundation under the project IP-2018-01-5591.

E-mail addresses: aneta@efos.hr (Antoaneta Klobučar), aklobucar@fsb.hr (Ana Klobučar)

() (i) This work is licensed under https://creativecommons.org/licenses/by/4.0/ 


\section{Introduction}

In the 4th century AD Constantine I (274 - 337 AD) ruled the Roman Empire. To defend the Empire against barbarians, he had to arrange Roman legions in a way that all strategically important places were protected with as low costs as possible.

If at least one Roman legion was stationed at a certain location, that location was considered to be secured. Unsecured locations, on the other hand, had no legions, but they had to be adjacent to at least one secured location. If an unsecured location was under attack, one could send a legion from some neighbouring secured location. But to avoid making that secured location unsecure, it had to have at least two legions itself. Maintaining of an army was expensive, so Constantine had to secure the Empire with as few legions as possible.

This historical background motivated Ian Stewart (1999) to suggest the new variant of graph domination known as Roman domination (RD). If we represent locations of the Empire as graph vertices and roads of the Empire as graph edges, the problem of defending the Roman Empire becomes a problem of graph domination. Double Roman domination (DRD) is stronger version in which we double protection.

There are many works dealing with Roman domination [8, 9, 13, 14], but only few about double Roman dominations. Foundations of DRD are set in [4]. In [3, 15, 16] we can find bounds on the DRD and the most recent work is [2]. For more details on Roman domination and double Roman domination and their variants see [5, 6, 7].

In this paper we determine exact values or upper and lower bounds for double Roman domination numbers on cardinal products of some graphs.

Apart from this introduction, the work is organized in the following way. In Section 2 we define dominating function on a graph $G$, Roman dominating function on $G$, double Roman dominating function on $G$ and on a cardinal product of graphs. Domination number, Roman domination number and double Roman domination number are defined and some basic relations among them are given.

In Section 3 we determine one upper and one lower bound for double Roman domination numbers on cardinal product of any two graphs. Then we determined the exact values of double Roman domination numbers of $P_{2} \times G$ for many types of graph $G$. Finally, the double Roman domination number is found for $P_{2} \times P_{n}, P_{3} \times P_{n}, P_{4} \times P_{n}$, while upper and lower bounds are given for $P_{5} \times P_{n}$ and $P_{6} \times P_{n}$.

In Section 4 we give a case study to determine the efficiency of double protection. We will simulate a battle between Romans and barbarians in the cases of double Roman domination and standard Roman domination.

\section{Definitions}

Dominating function (DF) on $G=(V, E)$ is a function $f: V \rightarrow\{0,1\}$ satisfying the condition that every vertex $u$ for which $f(u)=0$ is adjacent to at least one vertex $v$ for which $f(v)=1$. Depending on values of $f$, we get the ordered partition $\left(V_{0}, V_{1}\right)$ of $V$ where each vertex in $V_{0}$ is adjacent to at least one vertex in $V_{1}$. The set $V_{1}$ is called $a$ dominating set.

We have bijection between the set of all functions $f: V \rightarrow\{0,1\}$ and the set of all ordered partitions $\left(V_{0}, V_{1}\right)$. Thus we are allowed to write $f=\left(V_{0}, V_{1}\right)$. The weight of $f$ equals $w(f)=\sum_{v \in V} f(v)=0 \cdot\left|V_{0}\right|+1 \cdot\left|V_{1}\right|=\left|V_{1}\right|$. Of course, we will look for dominating functions with the minimum weight. This weight $\gamma(G)$ is called the domination 
number of $G$.

Further, Roman dominating function (RDF) on $G=(V, E)$ is a function $f: V \rightarrow$ $\{0,1,2\}$ satisfying the condition that every vertex $u$ for which $f(u)=0$ is adjacent to at least one vertex $v$ for which $f(v)=2$. Since this function also induces the ordered partition of $V$ with $V_{i}=\{v \in V: f(v)=i\}, i \in\{0,1,2\}$, we are allowed to write $f=\left(V_{0}, V_{1}, V_{2}\right)$. The set $V_{1} \cup V_{2}$ is called a Roman dominating set. The weight of an RDF $f$ equals $w(f)=\sum_{v \in V} f(v)=0 \cdot\left|V_{0}\right|+1 \cdot\left|V_{1}\right|+2 \cdot\left|V_{2}\right|=\left|V_{1}\right|+2\left|V_{2}\right|$. The minimum such weight $\gamma_{R}(G)$ is called the Roman domination number on $G$.

In Roman domination, one legion is required to defend any attacked area. Double Roman domination is a stronger version of Roman domination that doubles the protection by ensuring that any attack can be defended by at least two legions.

Finally, double Roman dominating function (DRDF) on $G=(V, E)$ is a function $f: V \rightarrow\{0,1,2,3\}$ if it satisfies the following conditions:

1. If $f(v)=0$, then the vertex $v$ has at least two neighbours in $V_{2}$ or one neighbour in $V_{3}$.

2. If $f(v)=1$, then the vertex $v$ has at least one neighbour in $V_{2} \cup V_{3}$,

where by $V_{i}$ we denote the set of vertices assigned with $i$ by the function $f$. The set $V_{1} \cup V_{2} \cup V_{3}$ is called a double Roman dominating set. The weight of a DRDF equals $w(f)=\sum_{v \in V} f(v)=\left|V_{1}\right|+2\left|V_{2}\right|+3\left|V_{3}\right|$.

Double Roman domination number $\gamma_{d R}(G)$ equals the minimum weight among all double Roman dominating functions on $G$. A double Roman dominating function on $G$ with weight $\gamma_{d R}(G)$ is called a $\gamma_{d R}$-function of $G$.

In Roman domination at most two Roman legions are deployed at any location. But as we will see in what follows, the ability to deploy three legions at a given location provides a level of defense that is both stronger and more flexible. Also, the additional security we get is usually greater than the additional costs.

Here we can see a real benefit of double Roman domination. In the example of the star graph $K_{1, n-1}$ (see Figure 1), it is obvious that $\gamma_{d R}\left(K_{1, n-1}\right)=3$. Note that this is only one more than $\gamma_{R}\left(K_{1, n-1}\right)=2$. So we doubled the defense of a graph (at least two legions against each attack) with an added cost of no more than $50 \%$ of the cost of defending against each attack with one legion.

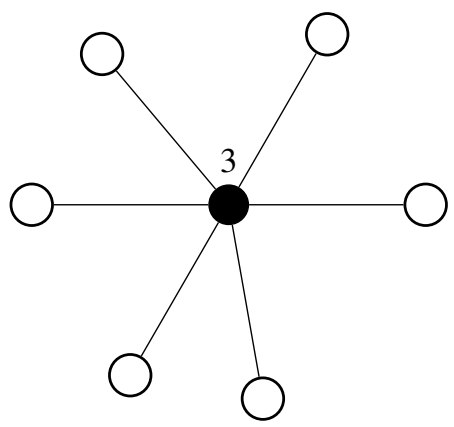

Figure 1: Double Roman domination on star graph. 
In [4], it is observed that $\gamma_{d R}(G) \leq 2\left|V_{1}\right|+3\left|V_{2}\right|$ for any RDF $f=\left(V_{0}, V_{1}, V_{2}\right)$. It is also proved a relation between domination and double Roman domination number of any graph $G$, i.e.

$$
2 \gamma(G) \leq \gamma_{d R}(G) \leq 3 \gamma(G),
$$

and a relation between Roman domination and double Roman domination number of any nontrivial connected graph $G$

$$
\gamma_{R}(G) \leq \gamma_{d R}(G) \leq 2 \gamma_{R}(G)
$$

Graphs where $\gamma_{d R}(G)=3 \gamma(G)$ are called double Roman graphs. There is an open problem to characterize such graphs. Double Roman trees are characterized in [1]. For more domination parameters and for the terminology see [10,11, 12].

In this paper we will consider double Roman domination number of cardinal product of graphs. For arbitrary graphs $G$ and $H$, the cardinal product of $G$ and $H$ is the graph $G \times H$ which satisfies the following:

1. Its vertex set is $V(G \times H)=V(G) \times V(H)$.

2. Two vertices $(g, h),\left(g^{\prime}, h^{\prime}\right) \in V(G \times H)$ are adjacent if and only if $g$ is adjacent to $g^{\prime}$ in $G$ and $h$ is adjacent to $h^{\prime}$ in $H$.

If $H \subset V(G)$ then $G[H]$ is the subgraph induced with $H$. The cardinal product of two paths $P_{m} \times P_{n}$ has two connected components. If the vertices of $P_{m}$ and $P_{n}$ are denoted by $\{1,2,3, \ldots, m\}$ and $\{1,2,3, \ldots, n\}$ respectively, then the component of $P_{m} \times$ $P_{n}$ containing the vertex $(1,1)$ will be denoted by $K_{1}$ and the other component by $K_{2}$. If at least one of the parameters $m$ or $n$ is even, the components $K_{1}$ and $K_{2}$ are isomorphic (see Figure 2). Otherwise, the component $K_{1}$ has one vertex more than the component $K_{2}$.
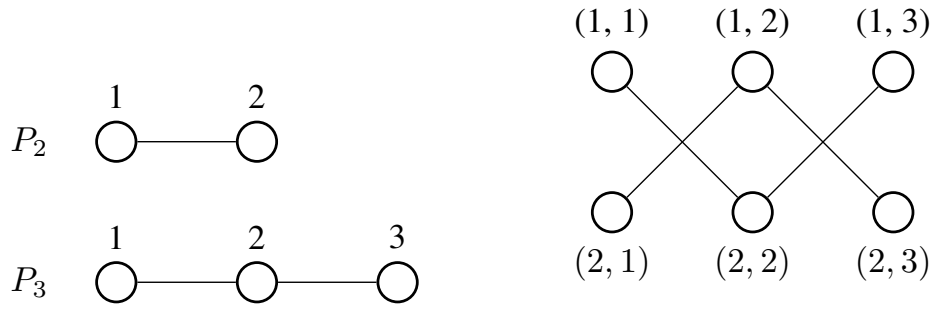

$$
P_{2} \times P_{3}
$$

Figure 2: $K_{1}=P_{2} \times P_{3}[\{(1,1),(2,2),(1,3)\}]$ and $K_{2}=P_{2} \times P_{3}[\{(2,1),(1,2),(2,3)\}]$.

\section{Specific values of double Roman domination numbers for cardinal products of graphs}

As for introduction, we will show here some basic results and bounds for double Roman domination. 
Remark 3.1. In [2] it is proved that

$$
\begin{aligned}
\gamma_{d R}\left(P_{n}\right) & = \begin{cases}3\left\lceil\frac{n}{3}\right\rceil, & n \equiv 0,2 \quad(\bmod 3) \\
3\left\lceil\frac{n}{3}\right\rceil-1, & n \equiv 1 \quad(\bmod 3)\end{cases} \\
& = \begin{cases}n, & n \equiv 0 \quad(\bmod 3) \\
n+1, & n \equiv 1,2 \quad(\bmod 3)\end{cases}
\end{aligned}
$$

and

$$
\gamma_{d R}\left(C_{n}\right)= \begin{cases}n, & n \equiv 0,2,3,4 \quad(\bmod 6) \\ n+1, & n \equiv 1,5 \quad(\bmod 6) .\end{cases}
$$

Observartion 3.2. For any graphs $G$ and $H$ of order $n$ and $m$

$$
\begin{aligned}
& \gamma_{d R}(G \times H) \geq\left\lceil\frac{3 m n}{\Delta(G) \Delta(H)+1}\right\rceil, \\
& \gamma_{d R}(G \times H) \leq 2 m n \frac{2+\ln ((1+\delta(G) \delta(H)) / 2)}{\delta(G) \delta(H)+1},
\end{aligned}
$$

where by $\Delta(G)(\delta(G))$ we denote the maximum (minimum) degree of all vertices on $G$.

Proof. In [16] it is proved that for any graph $G$ of order $n$ with maximum degree $\Delta(G) \geq 1$

$$
\gamma_{d R}(G) \geq\left\lceil\frac{3 n}{\Delta(G)+1}\right\rceil
$$

Further, it holds

$$
\Delta(G \times H)=\Delta(G) \cdot \Delta(H) .
$$

Combining two previous statements we get the lower bound. Next, in [14] it is proved that for cardinal product any graphs $G$ and $H$ of order $n$ and $m$

$$
\gamma_{R}(G \times H) \leq m n \frac{2+\ln ((1+\delta(G) \delta(H)) / 2)}{\delta(G) \delta(H)+1} .
$$

Then the statement follows from $\gamma_{d R}(G) \leq 2 \gamma_{R}(G)$.

Now we will calculate the exact values of double Roman domination numbers for cardinal products of some graphs.

Theorem 3.3. For any tree $T$ and any graph $G$ without cycles of odd length we have

$$
\begin{aligned}
& \gamma_{d R}\left(P_{2} \times T\right)=2 \gamma_{d R}(T)<\gamma_{d R}\left(P_{2}\right) \cdot \gamma_{d R}(T), \\
& \gamma_{d R}\left(P_{2} \times G\right)=2 \gamma_{d R}(G)<\gamma_{d R}\left(P_{2}\right) \cdot \gamma_{d R}(G) .
\end{aligned}
$$

Proof. The proof is trivial, since $P_{2} \times T$ and $P_{2} \times G$ consist of two disjoint copies of $T$ and $G$, respectively and $\gamma_{d R}\left(P_{2}\right)=3$.

Theorem 3.4. For the path $P_{2}$ and any odd cycle $C_{2 n+1}, n \geq 1$,

$$
\gamma_{d R}\left(P_{2} \times C_{2 n+1}\right)=4 n+2 .
$$


Proof. Note that the cardinal product of $P_{2}$ and $C_{2 n+1}$ is a cycle $C_{4 n+2}$. Namely, if we denote the vertices of $P_{2}$ with $a$ and $b$, and the vertices of $C_{2 n+1}$ with $1,2, \ldots, 2 n+1$, then the vertices of the product $P_{2} \times C_{2 n+1}$ are adjacent in this order: $(a, 1),(b, 2),(a, 3),(b, 4), \ldots$, $(a, 2 n+1),(b, 1),(a, 2), \ldots,(b, 2 n+1)$ and the last vertex $(b, 2 n+1)$ is adjacent to $(a, 1)$, which makes a cycle of length $2(2 n+1)=4 n+2$. Remark 3.1 implies that

$$
\gamma_{d R}\left(C_{4 n+2}\right)=4 n+2
$$

Definition 3.5. For a fixed $m, 1 \leq m \leq n$, the set $\left(P_{k}\right)_{m}=\{(i, m): i=1, \ldots, k\}$ is called a column of $P_{k} \times P_{n}$. Similary, for a fixed $l, 1 \leq l \leq k$, the set $\left(P_{n}\right)_{l}=\{(l, j)$ : $j=1, \ldots, n\}$ is called a row of $P_{k} \times P_{n}$.

Theorem 3.6. Let $n \geq 2$. Then

$$
\gamma_{d R}\left(P_{3} \times P_{n}\right)= \begin{cases}7, & n=3 \\ 2 n+2, & \text { otherwise }\end{cases}
$$

Proof. It is easy to see that $\gamma_{d R}\left(P_{3} \times P_{3}\right)=7$. Hence we assume $n \geq 4$. First we show that $\gamma_{d R}\left(P_{3} \times P_{n}\right) \leq 2 n+2$. Define $f: V\left(P_{3} \times P_{n}\right) \rightarrow\{0,1,2,3\}$ by $f((2,2))=$ $f((2, n-1))=3, f((2, j))=2$ for $j \in\{1, \ldots, n\}-\{2, n-1\}$ and $f(x)=0$ otherwise. Clearly $f$ is a double Roman dominating function on $P_{3} \times P_{n}$ of weight $2 n+2$ and so $\gamma_{d R}\left(P_{3} \times P_{n}\right) \leq 2 n+2$. To prove inverse inequality, let $f=\left(V_{0}, \emptyset, V_{2}, V_{3}\right)$ be a $\gamma_{d R}\left(P_{3} \times\right.$ $\left.P_{n}\right)$-function. Since the vertices $(2,2)$ and $(2, n-1)$ are strong support vertices, we have $(2,2),(2, n-1) \in V_{3}$. On the other hand, since $V_{2} \cup V_{3}$ is a dominating set of $P_{3} \times P_{n}$, we have $\left|V_{2} \cup V_{3}\right| \geq \gamma\left(P_{3} \times P_{n}\right)=n$ (see [11]). Thus we have $\gamma_{d R}\left(P_{3} \times P_{n}\right)=2\left|V_{2}\right|+3\left|V_{3}\right|=$ $2\left(\left|V_{2}\right|+\left|V_{3}\right|\right)+\left|V_{3}\right| \geq 2 n+2$. Thus $\gamma_{d R}\left(P_{3} \times P_{n}\right)=2 n+2$ for $n \geq 4$ and the proof is complete.

Theorem 3.7. Let $n \geq 2$. Then

$$
\gamma_{d R}\left(P_{4} \times P_{n}\right)=\left\{\begin{array}{lll}
3 n, & n \equiv 0 & (\bmod 4) \\
3 n+3, & n \equiv 1 & (\bmod 4) \\
3 n+2, & n \equiv 2 & (\bmod 4) \\
3 n+1, & n \equiv 3 & (\bmod 4)
\end{array}\right.
$$

Proof. First we show that

$$
\gamma_{d R}\left(P_{4} \times P_{n}\right) \leq\left\{\begin{array}{lll}
3 n, & n \equiv 0 & (\bmod 4) \\
3 n+3, & n \equiv 1 & (\bmod 4) \\
3 n+2, & n \equiv 2 & (\bmod 4) \\
3 n+1, & n \equiv 3 & (\bmod 4)
\end{array}\right.
$$

Since $P_{4} \times P_{n}$ consists of two isomorphic components, we consider only $K_{1}$ and we multiply the result by 2 .

Case 1: $n \equiv 0(\bmod 4)$. Define $f: V\left(K_{1}\right) \rightarrow\{0,1,2,3\}$ by $f((2,4 j+2))=$ $f((3,4 j+3))=3, j=0,1, \ldots,\left\lfloor\frac{n}{4}\right\rfloor-1$, and $f(x)=0$ otherwise. Clearly $f$ is a double Roman dominating function of weight $\frac{3 n}{2}$ on $K_{1}$ and so $\gamma_{d R}\left(P_{4} \times P_{n}\right) \leq 3 n$, for $n \equiv 0(\bmod 4)$. 
Case 2: $n \equiv 1(\bmod 4)$. Define $f: V\left(K_{1}\right) \rightarrow\{0,1,2,3\}$ by $f((2,4 j+2))=$ $f((3,4 j+3))=3, j=0,1, \ldots,\left\lfloor\frac{n}{4}\right\rfloor-1, f((2, n-1))=3$ and $f(x)=0$ otherwise. It can easily be seen that $f$ is a double Roman dominating function of weight $6\left(\frac{n-1}{4}\right)+3=\frac{3 n+3}{2}$ on $K_{1}$ and so $\gamma_{d R}\left(P_{4} \times P_{n}\right) \leq 3 n+3$, for $n \equiv 1(\bmod 4)$.

Case 3: $n \equiv 2(\bmod 4)$. Define $f: V\left(K_{1}\right) \rightarrow\{0,1,2,3\}$ by $f((2,4 j+2))=3$, $j=0,1, \ldots,\left\lfloor\frac{n}{4}\right\rfloor-1, f((3,4 j+3))=3, j=0,1, \ldots,\left\lfloor\frac{n}{4}\right\rfloor-2, f((3, n-1))=3$, $f((1, n-1))=f((4, n-4))=2$ and $f(x)=0$ otherwise. Hence $f$ is a double Roman dominating function of weight $6\left(\frac{n-2}{4}\right)+4=\frac{3 n+2}{2}$ on $K_{1}$ and so $\gamma_{d R}\left(P_{4} \times P_{n}\right) \leq 3 n+2$, for $n \equiv 2(\bmod 4)$.

Case 4: $n \equiv 3(\bmod 4)$. Define $f: V\left(K_{1}\right) \rightarrow\{0,1,2,3\}$ by $f((2,4 j+2))=$ $f((3,4 j+3))=3, j=0,1, \ldots,\left\lfloor\frac{n}{4}\right\rfloor-1, f((2, n-1))=3, f((4, n-1))=2$ and $f(x)=0$ otherwise. Therefore $f$ is a double Roman dominating function of weight $6\left(\frac{n-3}{4}\right)+5=\frac{3 n+1}{2}$ on $K_{1}$ and so $\gamma_{d R}\left(P_{4} \times P_{n}\right) \leq 3 n+1$, for $n \equiv 3(\bmod 4)$.

Proof of the minimality: In [16] is proved that if $G$ is a graph of order $n$ with maximum degree $\Delta(G) \geq 1$

$$
\gamma_{d R}(G) \geq\left\lceil\frac{3 n}{\Delta(G)+1}\right\rceil .
$$

The order of $P_{4} \times P_{n}$ is $4 n$ and $\Delta\left(P_{4} \times P_{n}\right)=4$. Therefore

$$
\gamma_{d R}\left(P_{4} \times P_{n}\right) \geq\left\lceil\frac{12 n}{5}\right\rceil=3 n .
$$

Let $n \equiv 0(\bmod 4)$. From the fact that $\gamma_{d R}\left(P_{4} \times P_{n}\right) \leq 3 n$ and (3.1), it follows

$$
\gamma_{d R}\left(P_{4} \times P_{n}\right)=3 n, \quad n \equiv 0 \quad(\bmod 4) .
$$

In more details, for this case each vertex from $V_{0}$ is double Roman dominated by only one vertex from $V_{3}$. Next, $V_{2}=\emptyset$, and $V_{3}$ is dominating set (see [11]). Also, on the last $n$-th column from $P_{4} \times P_{n}$ all vertices are from $V_{0}$ (see Figure 3 ).

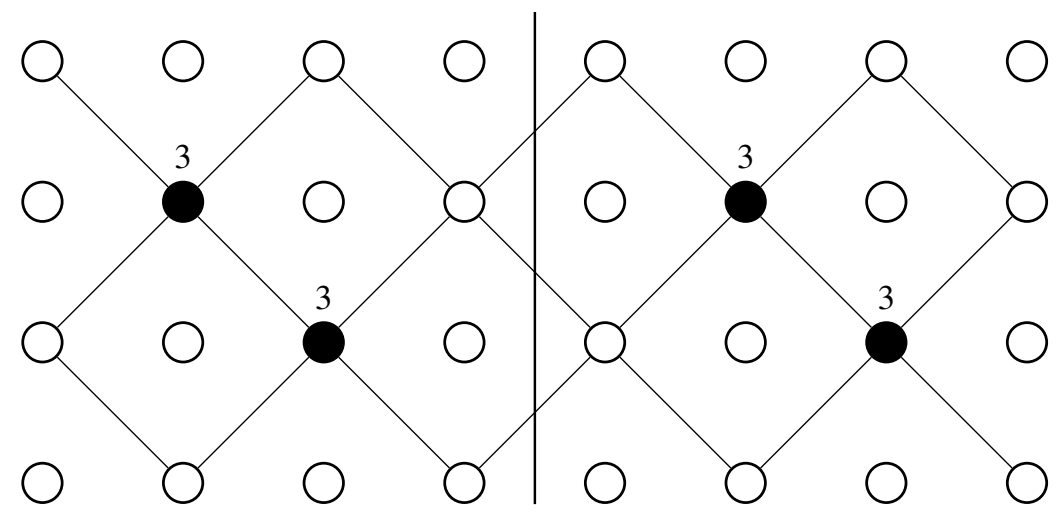

Figure 3: The function $f\left(V\left(K_{1}\right)\right)$ on $P_{4} \times P_{8}$.

Let $n \equiv 1(\bmod 4)$. Then from (3.1) on the first $n-1$ columns on $P_{4} \times P_{n}$ double Roman function $f$ has a weight at least $3(n-1)$. Further, if the function $f$ has the exactly 
weight $3 n-3$, then the vertex $(2, n-1) \in V_{0}$ (on $\left.K_{1}\right)$. But $(2, n-1)$ is strong suport vertex, so must be in $V_{3}$. The same situation is on $K_{2}$. It follows that $\gamma_{d R}\left(P_{4} \times P_{n}\right) \geq$ $3 n-3+6=3 n+3$. Hence,

$$
\gamma_{d R}\left(P_{4} \times P_{n}\right)=3 n+3, n \equiv 1 \quad(\bmod 4) .
$$

Let $n \equiv 2(\bmod 4)$. It is easy to see that $\gamma_{d R}\left(P_{4} \times P_{6}\right)=20$ (on each component $10)$ and that the last $4 \times 4$ block and $n^{\text {th }}$ and $(n-1)^{\text {th }}$ columns make a $4 \times 6$ block. It follows that on the last 6 columns we need at least weight 20 , and on the first $n-6$ columns $3(n-6)$. Then $\gamma_{d R}\left(P_{4} \times P_{n}\right) \geq 3 n-18+20=3 n+2$. So,

$$
\gamma_{d R}\left(P_{4} \times P_{n}\right)=3 n+2, n \equiv 2 \quad(\bmod 4) .
$$

Let $n \equiv 3(\bmod 4)$. Then on the first $n-3$ columns on $P_{4} \times P_{n}$ double Roman function $f$ has a weight at least $3(n-3)$. On the last 3 columns we need at least weight 5 on one, or 10 on both components giving $\gamma_{d R}\left(P_{4} \times P_{3}\right)=10$. It follows that $\gamma_{d R}\left(P_{4} \times P_{n}\right) \geq$ $3 n-9+10=3 n+1$. Therefore,

$$
\gamma_{d R}\left(P_{4} \times P_{n}\right)=3 n+1, n \equiv 3 \quad(\bmod 4) .
$$

For $P_{5} \times P_{n}$ and $P_{6} \times P_{n}$ from the formula

$$
2 \gamma(G) \leq \gamma_{d R}(G) \leq 3 \gamma(G),
$$

and [11] we have the following bounds:

$$
\begin{aligned}
2\left\{\begin{array}{ll}
n+2, & n=2,3,4 \\
11, & n=7 \\
\frac{4 n+6}{3}, & n \equiv 0,3 \quad(\bmod 6) \\
\frac{4 n+4}{3}, & n \equiv 2,5 \quad(\bmod 6) \\
\frac{4 n+8}{3}, & n \equiv 1,4 \quad(\bmod 6), n>7
\end{array} \quad \leq \gamma_{d R}\left(P_{5} \times P_{n}\right),\right. \\
\qquad \gamma_{d R}\left(P_{5} \times P_{n}\right) \leq 3 \begin{cases}n+2, & n=2,3,4 \\
11, & n=7 \\
\frac{4 n+6}{3}, & n \equiv 0,3 \quad(\bmod 6) \\
\frac{4 n+4}{3}, & n \equiv 2,5 \quad(\bmod 6) \\
\frac{4 n+8}{3} . & n \equiv 1,4 \quad(\bmod 6), n>7,\end{cases} \\
4\left(n-\left\lfloor\frac{n}{5}\right\rfloor\right) \leq \gamma_{d R}\left(P_{6} \times P_{n}\right) \leq 6\left(n-\left\lfloor\frac{n}{5}\right\rfloor\right) .
\end{aligned}
$$

\section{A case study}

In this section we simulate a battle between Romans and barbarians to test efficiency of the double protection versus the ordinary protection (standard Roman domination). Cardinal product $P_{4} \times P_{n}$ is used to model the battlefield, more precisely component $K_{1}$. We could use any other cardinal product of graphs, but we use $P_{4} \times P_{n}$ because of its convenience: it is large enough to have multiple outcomes, but not too large for visualization.

Instead of Romans and barbarians, we could have ambulances and patients or firefighters and fires. Ambulances would respond to medical emergencies and firefighters would 
extinguish fires in their local area. Position of hospitals and fire stations would correspond base vertices, respectively. We are still speaking about Romans and barbarians in order to conform with the usual terminology dealing with dominations. But as we can see, the whole situation has also some modern interpretations, which are more practical and more useful.

First, we will give some basic rules and restrictions to avoid exceptions. The following rules could be easily adapted to ambulances and patients, and to firefighters and fires.

- The simulation is organized in turns. The first turn is played by the barbarians.

- The simulation stops if all cities are destroyed by the barbarians or if all barbarians are defeated by legions and legions are returned to their base cities.

- The legions and the barbarian groups move only by one edge in each turn.

- The barbarian groups destroy an unprotected city if Romans do not send enough help in the next turn.

- If the barbarian group attacks a city with a legion, they fight immediately (no waiting for the next turn).

- The destroyed city stays destroyed, but both the legions and barbarian groups can pass through it.

- If the legions are outnumbered, they all die and no barbarian group dies. An analogous rule holds if the barbarians are outnumbered.

- If there is an equal number of legions and barbarian groups, Romans always win.

- Base cities defend only their direct neighbours.

- A base city does not send help to a neighbour if it cannot send enough help.

- At least one legion must stay in its base city.

- If a direct neighbour is secured, the legion returns to its base city.

- If a city is destroyed, the barbarian group moves to the closest undestroyed city. If there is more then one, then it moves randomly.

- Different barbarian groups move independently.

In the case of double Roman dominations, the initial number of Roman legions and their positions on the graph will be defined as for minimum double Roman domination sets in Theorem 3.7. In the case of standard Roman dominations, the layout of Roman legions will be defined as for minimal Roman domination sets [14], i.e. for $P_{4} \times P_{n}, n \equiv 0$ $(\bmod 6)$ and $K_{1}$ the minimal Roman domination set is:

$$
\begin{aligned}
& V_{1}=\left\{(1,6 j+5),(4,6 j+2): j=0,1, \ldots,\left\lfloor\frac{n}{6}\right\rfloor-1\right\} \quad \text { and } \\
& V_{2}=\left\{(2,6 j+2),(3,6 j+5): j=0,1, \ldots,\left\lfloor\frac{n}{6}\right\rfloor-1\right\} .
\end{aligned}
$$

Vertices with initial legions are called the base vertices or base cities. The initial number and placement of barbarian groups is arbitrary, but we will put at most 2 barbarian groups into one city. We do not want to destroy all cities in the very beginning.

We consider the placement of the barbarian groups as the first move done by barbarians. The next turn is on the legions. In each turn we have to check the state of each city i.e. the 
number of barbarian groups and legions and determine their next move. The number of legions and barbarian groups is fixed (it can only decay by turns).

Because on $P_{4} \times P_{n}$ we have two symmetrical components we will consider only $K_{1}$. On this component from Theorem 3.7, in the case of double Roman dominations, on the graph $P_{4} \times P_{12}$ we have 6 base cities and 3 legions in each of them. So 18 legions defend 24 cities. In case of standard Roman dominations, we have 8 base cities with total of 12 legions.

We have noticed that for $P_{4} \times P_{12}$ there exists a second layout for base vertices. It has also total sum of 12 legions, but they are placed differently while still satisfying Roman domination.

In Figure 4, Figure 5 and Figure 6, we see an initial placement for the standard and double protections.

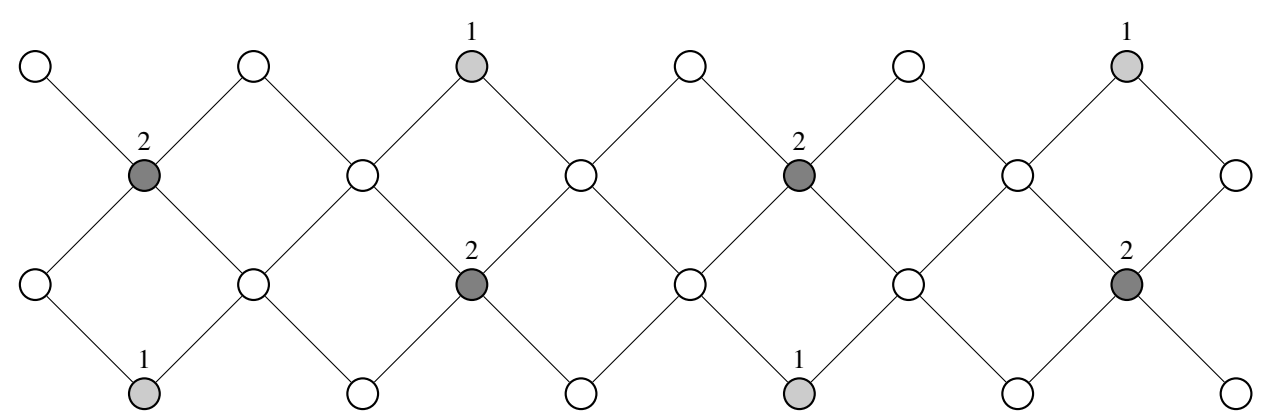

Figure 4: First version of initial placement of legions for $P_{4} \times P_{12}$ according to Roman dominating set with the lowest weight [14].

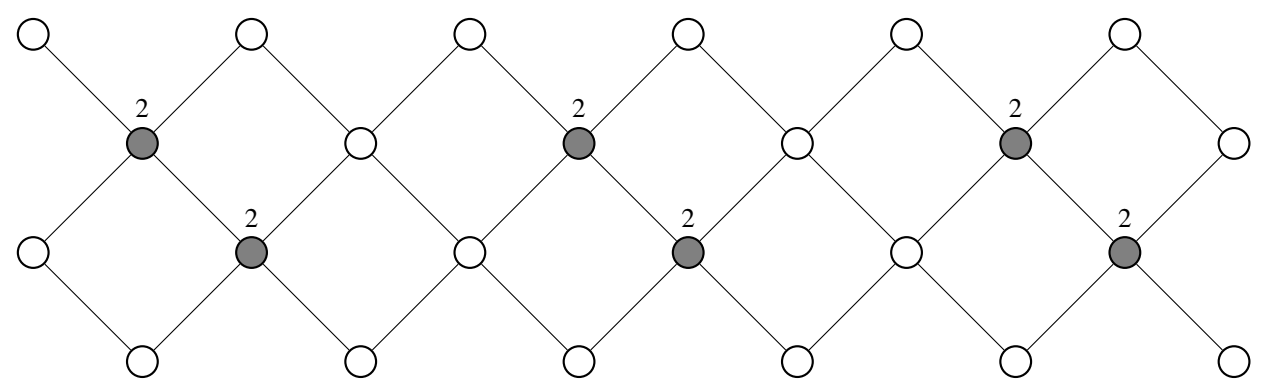

Figure 5: Second version of initial placement of legions for $P_{4} \times P_{12}$ according to Roman dominating set with the lowest weight.

Now we will test the both cases simultaneously. For the standard case we will take both layouts into consideration. Further, for a fixed number of barbarians, we will reproduce 30 random possibilities of attack for each case and measure number of destroyed cities and legions. Numbers of destroyed cities and legions will be represented with their arithmetic means.

First, we compare Roman dominating set with the first layout and double Roman dominating set. As shown in Table 1, Roman dominating set of 12 legions can survive at most 


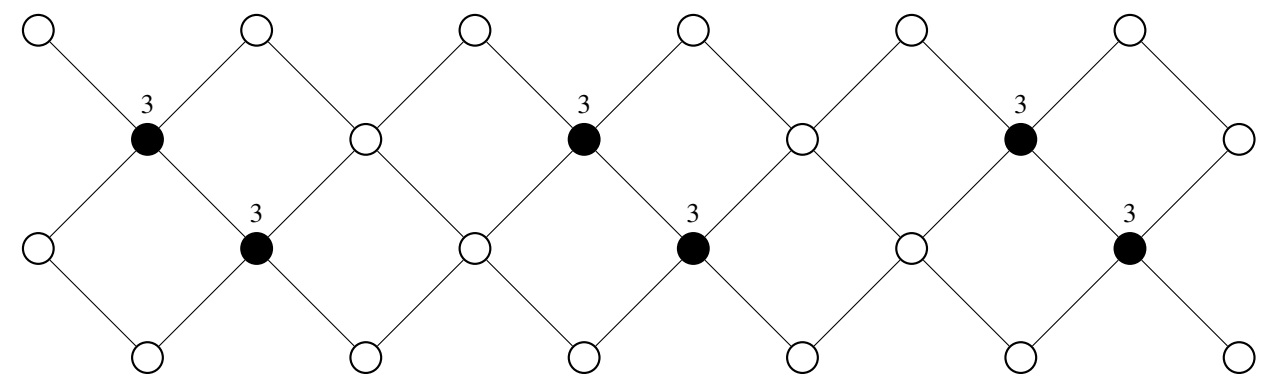

Figure 6: Initial placement of legions for $P_{4} \times P_{12}$ according to double Roman dominating set with the lowest weight.

25 barbarian groups according to our simulation, while double Roman dominating set of 18 legions can survive maximum 45 barbarion groups. So $50 \%$ more legions can survive $80 \%$ more barbarian groups on $P_{4} \times P_{12}$ which is efficiency increase of $30 \%$. Also, when all cities and legions are destroyed in case of Roman dominations, only $27 \%$ of cities and $9 \%$ of legions are destroyed for double Roman dominations.

Table 1: Average number of destroyed cities and legions at the end of the simulations.

\begin{tabular}{c|cc|cc|cc}
\multirow{2}{*}{$\begin{array}{c}\text { Barb. } \\
\text { legions }\end{array}$} & \multicolumn{2}{|c|}{ RD 1. layout } & \multicolumn{2}{c|}{ RD 2. layout } & \multicolumn{2}{c}{ DRD } \\
\cline { 2 - 7 } & $\begin{array}{c}\text { Destroyed } \\
\text { cities }\end{array}$ & $\begin{array}{c}\text { Destroyed } \\
\text { legions }\end{array}$ & $\begin{array}{c}\text { Destroyed } \\
\text { cities }\end{array}$ & $\begin{array}{c}\text { Destroyed } \\
\text { legions }\end{array}$ & $\begin{array}{c}\text { Destroyed } \\
\text { cities }\end{array}$ & $\begin{array}{c}\text { Destroyed } \\
\text { legions }\end{array}$ \\
\hline \hline 10 & 6.67 & 1.46 & 3.70 & 0.50 & 0.90 & 0.10 \\
20 & 20.66 & 9.13 & 15.87 & 6.13 & 4.53 & 0.73 \\
25 & 23.63 & 11.63 & 20.97 & 9.33 & 6.56 & 1.66 \\
29 & 24 & 12 & 23.53 & 11.53 & 9.80 & 3.67 \\
30 & 24 & 12 & 24 & 12 & 10.53 & 4.40 \\
40 & 24 & 12 & 24 & 12 & 21.53 & 15.2 \\
45 & 24 & 12 & 24 & 12 & 23.83 & 17.76 \\
46 & 24 & 12 & 24 & 12 & 24 & 18
\end{tabular}

Second, we compare Roman dominating set with the second layout and double Roman dominating set. Now Roman dominating set of 12 legions can survive at most 29 barbarian groups. So $50 \%$ more legions can survive $55 \%$ more barbarion groups. The increase in efficiency is considerably less than for the first layout.

What is common to the second layout of Roman dominating set and double Roman dominating set is that base cities are closer and bigger. It means that it is better to have few base cities with higher number of legions than more base cities with smaller number.

\section{Conclusion}

In this paper bounds for double Roman domination numbers for the cardinal product of any two graphs are given. Also, the exact values are given for the cardinal product of $P_{2}$ with any graph, for $P_{3} \times P_{n}$ and for $P_{4} \times P_{n}$. Furthermore, upper and lower bounds for double Roman domination numbers of $P_{5} \times P_{n}$ and $P_{6} \times P_{n}$ are given. 
We have also created a case study in wich we have compared Roman domination and double Roman domination on a cardinal product of graphs. The case study has confirmed that double Roman domination is more efficient because for small cost we can multiple protection.

Double Roman domination can be useful even today, not only in military sense. For example, in unsecure parts of a town, where calls for police are common, there should be at least three teams ready to go out after a call. So, when two teams are gone, the thmining team can react to some new call. Such services already exist in emergency medical stations.

\section{ORCID iDs}

Ana Klobučar (iD https://orcid.org/0000-0002-0260-5439

\section{References}

[1] H. Abdollahzadeh Ahangar, J. Amjadi, M. Atapour, M. Chellali and S. M. Sheikholeslami, Double Roman trees, Ars Combin. 145 (2019), 173-183.

[2] H. Abdollahzadeh Ahangar, M. Chellali and S. M. Sheikholeslami, On the double Roman domination in graphs, Discrete Appl. Math. 232 (2017), 1-7, doi:10.1016/j.dam.2017.06.014.

[3] J. Amjadi, S. Nazari-Moghaddam, S. M. Sheikholeslami and L. Volkmann, An upper bound on the double Roman domination number, J. Comb. Optim. 36 (2018), 81-89, doi:10.1007/ s10878-018-0286-6.

[4] R. A. Beeler, T. W. Haynes and S. T. Hedetniemi, Double Roman domination, Discrete Appl. Math. 211 (2016), 23-29, doi:10.1016/j.dam.2016.03.017.

[5] M. Chellali, N. Jafari Rad, S. M. Sheikholeslami and L. Volkmann, Roman domination in graphs, in: T. W. Haynes, S. T. Hedetniemi and M. A. Henning (eds.), Topics in Domination in Graphs, Springer, Cham, volume 64 of Developments in Mathematics, pp. 365-409, 2020, doi:10.1007/978-3-030-51117-3_11.

[6] M. Chellali, N. Jafari Rad, S. M. Sheikholeslami and L. Volkmann, Varieties of Roman domination, in: T. W. Haynes, S. T. Hedetniemi and M. A. Henning (eds.), Structures of Domination in Graphs, Springer, Cham, volume 66 of Developments in Mathematics, 2020.

[7] M. Chellali, N. Jafari Rad, S. M. Sheikholeslami and L. Volkmann, Varieties of Roman domination II, AKCE Int. J. Graphs Comb. (2020), doi:10.1016/j.akcej.2019.12.001.

[8] E. J. Cockayne, P. A. Dreyer, Jr., S. M. Hedetniemi and S. T. Hedetniemi, Roman domination in graphs, Discrete Math. 278 (2004), 11-22, doi:10.1016/j.disc.2003.06.004.

[9] O. Favaron, H. Karami, R. Khoeilar and S. M. Sheikholeslami, On the Roman domination number of a graph, Discrete Math. 309 (2009), 3447-3451, doi:10.1016/j.disc.2008.09.043.

[10] T. W. Haynes, S. T. Hedetniemi and P. J. Slater, Fundamentals of Domination in Graphs, volume 208 of Monographs and Textbooks in Pure and Applied Mathematics, Marcel Dekker, New York, 1998, doi:10.1201/9781482246582.

[11] A. Klobučar, Domination numbers of cardinal products, Math. Slovaca 49 (1999), 387-402, http://hdl.handle.net/10338.dmlcz/128761.

[12] A. Klobučar, Domination numbers of cardinal products $P_{6} \times P_{n}$, Math. Commun. 4 (1999), 241-250, https://hrcak.srce.hr/877.

[13] A. Klobučar and I. Puljić, Some results for Roman domination number on cardinal product of paths and cycles, Kragujevac J. Math. 38 (2014), 83-94, doi:10.5937/kgjmath1401083k. 
A. Klobučar and A. Klobučar: Properties of double Roman domination on cardinal products ... 349

[14] A. Klobučar and I. Puljić, Roman domination number on cardinal product of paths and cycles, Croat. Oper. Res. Rev. CRORR 6 (2015), 71-78, doi:10.17535/crorr.2015.0006.

[15] D. A. Mojdeh, A. Parsain and I. Masoumi, Bounds on double Roman domination number of graphs, in: Proceedings of the 2nd International Conference on Combinatorics, Cryptography and Computation (I4C2017), 2017 pp. 429-434, http: / i 4c.iust.ac.ir/2017/ UPL/Paper17/i4c17-1061.pdf.

[16] L. Volkmann, Double Roman domination and domatic numbers of graphs, Commun. Comb. Optim. 3 (2018), 71-77, doi:10.22049/cco.2018.26125.1078. 